\title{
Características físicas e químicas de morango processado minimamente e conservado sob refrigeração e atmosfera controlada
}

\author{
Physical and chemical characteristics of minimally processed strawberries \\ stored under refrigeration and controled atmospheres
}

\begin{abstract}
Ingrid Vieira Machado de MORAES ${ }^{1}$, Sérgio Agostinho $\mathrm{CENCI}^{2}$, Benedito Carlos BENEDETTI ${ }^{3 *}$, Alexandra Mara Goulart Nunes MAMEDE ${ }^{4}$, Antônio Gomes SOARES ${ }^{2}$, Henriqueta Talita Guimarães BARBOZA²
\end{abstract}

\section{Resumo}

Morangos cv. Oso Grande foram processados minimamente e mantidos em três diferentes atmosferas: $3 \% \mathrm{O}_{2}+10 \% \mathrm{CO}_{2}$ (balanço $\mathrm{N}_{2}$ ), $3 \% \mathrm{O}_{2}+15 \% \mathrm{CO}_{2}$ (balanço $\mathrm{N}_{2}$ ) e atmosfera ambiente (controle), a 5 e $10{ }^{\circ} \mathrm{C}$, durante 7 dias, com o objetivo de avaliar suas características físicas e químicas ao longo do armazenamento. Os frutos foram avaliados nos dias 0,3 e 7 quanto à perda de massa, firmeza, $\mathrm{pH}$, acidez titulável, sólidos solúveis, açúcares (sacarose, frutose e glicose) e teor de antocianinas totais. Os dados foram submetidos à análise de variância (ANOVA), e as médias comparadas estatisticamente pelo teste de Diferença Mínima Significativa (LSD), a 5\% de probabilidade. Verificou-se que o aumento da temperatura, de 5 para $10{ }^{\circ} \mathrm{C}$, não influenciou de forma significativa as variáveis físicas e químicas avaliadas. A utilização das atmosferas contendo $3 \% \mathrm{O}_{2}+10 \% \mathrm{CO}_{2}$ e $3 \% \mathrm{O}_{2}+15 \% \mathrm{CO}_{2}$ foi importante para a manutenção da qualidade dos morangos processados minimamente, pois mantiveram melhor firmeza em relação à atmosfera ambiente, e foram mais efetivas no controle da perda de massa dos frutos. Todavia, os frutos processados minimamente mantidos nestas atmosferas apresentaram menor teor de antocianinas que os mantidos em atmosfera ambiente.

Palavras-chave: Fragaria $x$ Ananassa Duch; processamento mínimo; controle atmosférico; armazenamento refrigerado; qualidade.

\begin{abstract}
Strawberries cv. Oso Grande were minimally processed and kept in three different atmospheres: $3 \% \mathrm{O}_{2}+10 \% \mathrm{CO}_{2}\left(\mathrm{~N}_{2}\right.$ balance), $3 \% \mathrm{O}_{2}+15 \% \mathrm{CO}_{2}\left(\mathrm{~N}_{2}\right.$ balance) and ambient atmosphere (control), at 5 and $10{ }^{\circ} \mathrm{C}$, during 7 days, with the evaluation of their physical and chemical characteristics during storage. Fruits were evaluated at 0,3 and 7 days for weight loss, firmness, $\mathrm{pH}$, titratable acidity, soluble solids, sugar content (sucrose, fructose and glucose) and total anthocyanins. Data were subjected to analysis of variance (ANOVA) and means were compared by the Least Significance Difference (LSD) test (5\%). It was verified that shifting the temperature from 5 to $10^{\circ} \mathrm{C}$ had no significant effect on the physical and chemical variables analyzed. The utilization of atmospheres $3 \% \mathrm{O}_{2}+10 \% \mathrm{CO}_{2}$ and $3 \% \mathrm{O}_{2}+15 \% \mathrm{CO}_{2}$ was important for quality maintenance of fresh-cut strawberries. They better preserved firmness in relation to ambient atmosphere, and had a positive effect on the control of fruit weight loss. However, fresh-cut fruits kept under those atmospheres showed a lower content of anthocyanins when compared to fruits stored in ambient conditions.
\end{abstract}

Keywords: Fragaria $x$ Ananassa Duch; minimal processing; atmospheric control; refrigerated storage; quality.

\section{Introdução}

Durante muito tempo os esforços da pesquisa em tecnologia de alimentos foram centrados na conservação de matérias-primas in natura, com o objetivo de prolongar sua vida útil. As condicionantes atuais da produção de refeições colocam uma nova necessidade, a de produtos prontos para o consumo. Existe uma tendência mundial para usar alimentos cada vez mais naturais, valorizando o sabor original dos produtos, na qual o consumidor, disposto a pagar mais pela qualidade, apresenta um nível de exigência cada vez maior. É neste cenário que surgem os alimentos processados minimamente, que unem a praticidade e a conveniência, proporcionando uma economia de tempo no preparo dos alimentos.
O processamento mínimo é definido como qualquer alteração física causada em frutas e hortaliças e que mantém o estado fresco destes produtos (INTERNATIONAL FRESH-CUT PRODUCE ASSOCIATION, 1999). Inclui as operações de seleção, limpeza, lavagem, descascamento, corte, sanificação, centrifugação e embalagem, ou seja, operações que não afetem suas características sensoriais e agreguem valor aos mesmos, resultando em produtos naturais e práticos, cujo preparo e consumo requer menos tempo, atendendo às exigências da vida moderna (DAMASCENO; STAMFORD; ALVES, 2001).

Recebido para publicação em 16/7/2007

Aceito para publicação em 12/2/2008 (001726)

1 Embrapa Agroindústria Tropical, CP 13761, CEP 60511-110, Fortaleza - CE, Brasil, E-mail: ingrid@cnpat.embrapa.br

2 Embrapa Agroindústria de Alimentos, Av. das Américas, 29.501, Guaratiba, CEP 23020-470, Rio de Janeiro - RJ, Brasil

${ }^{3}$ Faculdade de Engenharia Agrícola, Universidade Estadual de Campinas - Unicamp, Cidade Universitária Zeferino Vaz, CP 6011, CEP 13083-875, Campinas - SP, Brasil,

E-mail: benedeti@agr.unicamp.br

${ }^{4}$ Departamento de Ciência dos Alimentos, Universidade Federal de Lavras - UFLA, Lavras - MG, Brasil

${ }^{*}$ A quem a correspondência deve ser enviada 
O morango é uma hortaliça de clima temperado e tem apelo peculiar por sua coloração vermelha brilhante, odor característico, textura macia e sabor levemente acidificado. Dada a sua grande demanda na culinária nacional e internacional, é desejável o desenvolvimento de tecnologia para o seu processamento mínimo. Embora o processamento seja mínimo e a tecnologia aparentemente simples, há uma série de cuidados para que os produtos processados minimamente apresentem $o$ frescor esperado, sejam seguros para a saúde e tenham uma vida útil comercialmente viável. O processamento mínimo aumenta a perecibilidade do produto, dado o aumento da atividade metabólica e da descompartimentalização de enzimas e substratos, podendo resultar em escurecimento, perda de firmeza e desenvolvimento de sabores e odores estranhos (ROLLE; CHISM, 1987; WATADA; ABE; YAMUCHI, 1990).

A refrigeração é considerada o meio mais efetivo para estender a vida útil de frutas e hortaliças processadas minimamente (HONÓRIO; MORETTI, 2001). Temperaturas de refrigeração contribuem para reduzir a atividade microbiana e as alterações químicas e enzimáticas do próprio vegetal. Isso mantém a qualidade do produto e aumenta a segurança para o consumidor (BRACKETT, 1987; BRECHT et al., 2003). A redução da concentração de $\mathrm{O}_{2}$ e/ou o aumento da concentração de $\mathrm{CO}_{2}$ na atmosfera em contato com as frutas e hortaliças in natura ou processadas minimamente podem diminuir as suas taxas respiratórias e a produção de etileno. Desta maneira, pode haver inibição ou diminuição das reações enzimáticas, reduzindo desordens fisiológicas e evitando várias alterações metabólicas que resultam em deterioração pós-colheita (LANA; FINGER, 2000; SOLIVA-FORTUNY; MARTÍN-BELLOSO, 2003). Em geral, o uso da refrigeração, associado a um controle da atmosfera, é responsável por manter características qualitativas importantes em morangos, como firmeza, sólidos solúveis, acidez titulável e frescor, podendo ainda reduzir a incidência de fungos (AGAR; STREIF; BANGERTH, 1997).

O presente trabalho teve como objetivo avaliar, por meio de análises físicas e químicas, a qualidade de morangos processados minimamente mantidos sob refrigeração e atmosfera controlada.

\section{Material e métodos}

\subsection{Matéria-prima}

Morangos da cultivar Oso Grande foram colhidos no período da manhã, em cultivo comercial localizado no município de Pouso Alegre - MG, e selecionados por tamanho $( \pm 25-30 \mathrm{~mm})$ e cor (4/4 de coloração vermelha) desejados para o processamento mínimo. Os morangos foram transportados em veículo com temperatura em torno de $22^{\circ} \mathrm{C}$ para o Laboratório de Póscolheita da Embrapa Agroindústria de Alimentos, localizada no município do Rio de Janeiro. Os frutos foram mantidos em câmara fria a $5^{\circ} \mathrm{Ce} 85 \%$ de umidade relativa (UR) por aproximadamente 10 horas até o início do processamento no dia seguinte. O tempo total entre a colheita e o início do processamento foi de aproximadamente 24 horas.

\subsection{Processamento mínimo do morango}

Realizou-se o corte do cálice/pedúnculo dos morangos, sanificação por imersão em água com temperatura variando de 5 a $8{ }^{\circ} \mathrm{C} / 10$ minutos, contendo $150 \mathrm{ppm}$ de cloro ativo, enxágüe por imersão em água com temperatura variando de $5 \mathrm{a}$ $8{ }^{\circ} \mathrm{C} / 5$ minutos, contendo $5 \mathrm{ppm}$ de cloro ativo. Em seguida, foram colocados em escorredores para a drenagem da água em excesso. Depois de secos, os morangos foram pesados, acondicionados em embalagem de polietileno tereftalato (PET) e mantidos sob atmosfera controlada durante 7 dias, a 5 e a $10^{\circ} \mathrm{C}$ e $85 \%$ UR. O controle da atmosfera foi feito em microcâmaras, localizadas dentro de câmaras frias, sendo estabelecidas as seguintes composições atmosféricas: $\mathrm{AC} 1-3 \%$ $\mathrm{O}_{2}+10 \% \mathrm{CO}_{2}$ (balanço $\mathrm{N}_{2}$ ), $\mathrm{AC} 2-3 \% \mathrm{O}_{2}+15 \% \mathrm{CO}_{2}$ (balanço $\mathrm{N}_{2}$ ), AA - Atmosfera Ambiente (controle). Cada parcela experimental consistiu de uma embalagem com aproximadamente $300 \mathrm{~g}$ de morango processado minimamente. Para cada tempo de avaliação previsto, as amostras de cada tratamento foram armazenadas em triplicata.

\subsection{Análises físico-químicas}

Os frutos foram avaliados nos dias 0, 3 e 7 do armazenamento. Para as análises de $\mathrm{pH}$, acidez titulável, teor de sólidos solúveis, teor de açúcares e de antocianinas totais, foi obtido um extrato por meio de trituração e homogeneização em blender dos morangos que compunham a unidade experimental (embalagem com $300 \mathrm{~g}$ de morango).

\section{Perda de massa}

Obtida por meio da diferença entre as pesagens das embalagens contendo o produto em cada intervalo de tempo e o tempo zero, sendo o resultado expresso em porcentagem.

\section{Firmeza}

Medida por meio do equipamento Texture Analyser, modelo TA-Hdi (Stable Micro Systems, Surrey, Reino Unido), usando célula de carga de $5 \mathrm{~kg}$. O diâmetro da ponta de teste utilizada foi de $5 \mathrm{~mm}$, com uma velocidade de penetração de $0,16 \mathrm{~mm} \mathrm{~s}^{-1}$ e $3 \mathrm{~mm}$ de deformação da polpa. Dez morangos processados minimamente de cada repetição foram cortados ao meio e foi realizada uma medida na região equatorial de cada metade. Os resultados foram expressos em Newtons $(\mathrm{N})$.

\section{$p H$}

Determinado por potenciometria em titulador automático Titroline 96 Schott (SCHOTT AG, Mainz, Alemanha), segundo a ISO 1842 (ISO, 1991).

\section{Acidez total titulável (ATT)}

Determinada por titulometria com $\mathrm{NaOH} 0,1 \mathrm{~N}$ até pH 8,1 em titulador automático Titroline 96 Schott (SCHOTT AG, Mainz, Alemanha), segundo a ISO 750 (ISO, 1998). Os resultados foram expressos em mg ácido cítrico $100 \mathrm{~g}^{-1}$. 


\section{Sólidos solúveis (SS)}

Determinados por meio da leitura em refratômetro digital Atago PR-101 (Atago Co. Ltd, Tokyo, Japão), com os resultados expressos em ${ }^{\circ}$ Brix, segundo a ISO 2173 (ISO, 1978).

\section{Teor de açúcares (glicose, frutose e sacarose)}

Determinados segundo Macrae (1998), por separação cromatográfica da amostra em coluna de Fase Reversa e conseqüente determinação da concentração dos açúcares por Cromatrografia Líquida de Alta Eficiência (CLAE), utilizando método de padronização externa. As seguintes condições cromatográficas foram utilizadas: Coluna Amino $30 \mathrm{~cm}$ x 4,6 mm (High Performance Carbohydrate), fase móvel Acetonitrila 75\% em água com fluxo de $1,4 \mathrm{~mL} / \mathrm{min}$ e detector índice de refração com temperatura interna de $45^{\circ} \mathrm{C}$. Os resultados foram expressos em g. $100 \mathrm{~g}^{-1}$.

\section{Teor de antocianinas totais}

Determinado por colorimetria, por meio de extração do pigmento com solução EtOH-HCl, concentração 90:10 v/v (etanol $95 \%$ acidificado com 1,5 N HCl, pH da solução extratora igual a 1,0) e leitura em Espectrofotômetro de UV-Visível SPECORD 205 no comprimento de onda de $520 \mathrm{~nm}$, segundo método de Spayd e Morris (1981) e Sistrunk e Morris (1978), baseados no método de Fuleki e Francis (1968), sendo os resultados expressos em mg pelargonidina 3-glicosídeo $(100 \mathrm{~g})^{-1}$ de morango fresco, que foi calculado através da seguinte Equação 1:

$\mathrm{AT}=\left(\mathrm{Abs}_{520} \mathrm{x}\right) /\left(\mathrm{E}_{1 \mathrm{~cm}}{ }^{1 \%} \mathrm{xM}\right)$

em que:

$\mathrm{AT}=$ Teor de antocianinas totais;

$\mathrm{Abs}_{520}=$ Absorbância da amostra a $520 \mathrm{~nm}$;

$\mathrm{V}=$ Volume aferido $(\mathrm{mL})$;

$\mathrm{E}_{1 \mathrm{~cm}}{ }^{1 \%}=$ Coeficiente de extinção da pelargonidina 3-glicosídeo a $1 \%$; e

$\mathrm{M}=$ Massa da amostra (g).

\subsection{Análise estatística}

O delineamento estatístico foi inteiramente casualizado, com 18 tratamentos provenientes de um fatorial $3 \times 2 \times 3$, sendo 3 atmosferas, 2 temperaturas e 3 tempos de avaliação, com 3 repetições. Os dados foram submetidos à análise de variância (ANOVA) e as médias comparadas pelo teste LSD a $5 \%$ de probabilidade.

\section{Resultados e discussão}

\subsection{Perda de massa}

Foram observadas diferenças significativas para os fatores atmosfera, tempo de armazenamento e para as interações temperatura-tempo e atmosfera-tempo $(\mathrm{p}<0,05)$. A temperatura de armazenamento, isolada, não afetou significativamente essa variável. A perda de massa foi significativamente maior para os frutos mantidos sob AA, em ambas as temperaturas. Os frutos mantidos nas atmosferas $\mathrm{AC} 1 \mathrm{e} \mathrm{AC} 2$ não diferiram significativamente entre si em nenhum dos períodos avaliados, tanto a $5^{\circ} \mathrm{C}$ quanto a $10^{\circ} \mathrm{C}$ (Tabela 1$)$.

Observou-se que os frutos mantidos sob AA tiveram perda de massa significativa ao longo do armazenamento, e cada período diferiu significativamente entre si, nas duas temperaturas avaliadas. Adicionalmente, observou-se que no $7^{\circ}$ dia de armazenamento a perda de massa dos frutos mantidos sob AA, em ambas as temperaturas, foi significativamente maior que as perdas dos demais tratamentos. Não houve perda significativa de massa nos frutos mantidos nas atmosferas AC1 e AC2 do $3^{\circ}$ para o $7^{\circ}$ dia de armazenamento quando mantidos a $5^{\circ} \mathrm{C}$, contudo, a $10^{\circ} \mathrm{C}$ houve perda de massa significativa nesse mesmo período nos frutos mantidos sob ACl. A perda de massa, no $7^{\circ}$ dia de armazenamento, para os morangos mantidos sob AA foi de $6,6 \%$ a $5{ }^{\circ} \mathrm{C}$ e de $7,7 \%$ a $10^{\circ} \mathrm{C}$. Já para os frutos mantidos nas atmosferas $\mathrm{AC} 1$ e $\mathrm{AC} 2$ os valores foram, respectivamente, 1,1 e $0,9 \%$ a $5{ }^{\circ} \mathrm{C}$ e 1,33 e $1,10 \%$ a $10{ }^{\circ} \mathrm{C}$.

A grande perda de massa ocorrida nos frutos processados minimamente e armazenados sob AA pode ser explicada pelo acelerado metabolismo dos frutos deixados nessa condição, em virtude da alta disponibilidade ao $\mathrm{O}_{2}$, com conseqüente aumento da transpiração pelo tecido vegetal.

Segundo Ronque (1998), a percentagem máxima de perda de água aceitável para a comercialização do morango é de $6 \%$ de seu peso na colheita. Acima deste patamar, o morango torna-se inaceitável para a comercialização. Sendo assim, verificou-se neste experimento que somente os frutos mantidos nas atmosferas AC1 e AC2 encontravam-se comercialmente aceitáveis após 7 dias de armazenamento.

\subsection{Firmeza}

Foram observadas diferenças significativas para os fatores atmosfera, tempo de armazenamento e para a interação entre eles $(\mathrm{p}<0,05)$. A temperatura de armazenamento, de maneira

Tabela 1. Perda de massa e variação da firmeza em morangos processados minimamente e mantidos sob atmosfera controlada a 5 e $10^{\circ} \mathrm{C}$ e $85 \%$ UR.

\begin{tabular}{|c|c|c|c|c|c|c|}
\hline \multirow{3}{*}{$\begin{array}{l}\text { Tempo de } \\
\text { armazenamento } \\
\text { (dias) }\end{array}$} & \multicolumn{6}{|c|}{ Tratamentos } \\
\hline & \multicolumn{2}{|c|}{$\mathrm{AC} 1$} & \multicolumn{2}{|c|}{$\mathrm{AC} 2$} & \multicolumn{2}{|c|}{ AA } \\
\hline & $5^{\circ} \mathrm{C}$ & $10^{\circ} \mathrm{C}$ & $5^{\circ} \mathrm{C}$ & $10^{\circ} \mathrm{C}$ & $5^{\circ} \mathrm{C}$ & $10^{\circ} \mathrm{C}$ \\
\hline & \multicolumn{6}{|c|}{ Perda de massa (\%) } \\
\hline 0 & $0,00^{\mathrm{Ab}}$ & $0,00^{\mathrm{Ab}}$ & $0,00^{\mathrm{Ab}}$ & $0,00^{\mathrm{Ab}}$ & $0,00^{\mathrm{Ac}}$ & $0,00^{\mathrm{Ac}}$ \\
\hline 3 & $0,91^{\mathrm{Ba}}$ & $0,23^{\mathrm{Bb}}$ & $0,67^{\mathrm{Ba}}$ & $0,45^{\mathrm{Bab}}$ & $3,75^{\mathrm{Ab}}$ & $2,62^{\mathrm{Ab}}$ \\
\hline \multirow[t]{2}{*}{7} & $1,10^{\mathrm{Ba}}$ & $1,33^{\mathrm{Ba}}$ & $0,91^{\mathrm{Ba}}$ & $1,10^{\mathrm{Ba}}$ & $6,60^{\mathrm{Aa}}$ & $7,70^{\mathrm{Aa}}$ \\
\hline & \multicolumn{6}{|c|}{ Firmeza $(\mathrm{N})$} \\
\hline 0 & $1,46^{\mathrm{Aa}}$ & $1,46^{\mathrm{Aa}}$ & $1,46^{\mathrm{Aa}}$ & $1,46^{\mathrm{Aa}}$ & $1,46^{\mathrm{Aa}}$ & $1,46^{\text {Aa }}$ \\
\hline 3 & $1,58^{\mathrm{ABa}}$ & $1,61^{\mathrm{Aa}}$ & $1,62^{\mathrm{Aa}}$ & $1,53^{\mathrm{ABa}}$ & $1,39^{\mathrm{Ba}}$ & $1,57^{\mathrm{ABa}}$ \\
\hline 7 & $1,38^{\mathrm{Ba}}$ & $1,28^{\mathrm{BCa}}$ & $1,62^{\mathrm{Aa}}$ & $1,36^{\mathrm{Ba}}$ & $1,11^{\mathrm{Cb}}$ & $1,15^{\mathrm{Cb}}$ \\
\hline
\end{tabular}

Médias seguidas pela mesma letra, maiúscula na horizontal e minúscula na vertical, são estatisticamente iguais pelo teste de LSD a $5 \%$ de significância. ACl $=3 \% \mathrm{O}_{2}+10 \% \mathrm{CO}_{2}$; $\mathrm{AC2}=3 \% \mathrm{O}_{2}+15 \% \mathrm{CO}_{2} ;$ e AA = atmosfera ambiente (controle). 
isolada e semelhante ao ocorrido com a perda de massa, também não afetou significativamente esta variável (Tabela 1).

Em geral, os frutos mantidos nas atmosferas AC1 e AC2 apresentaram firmeza superior aos mantidos sob AA. No $3^{\circ}$ dia de armazenamento a $5^{\circ} \mathrm{C}$, houve diferença significativa entre os frutos mantidos nas atmosferas AC2 $(1,62 \mathrm{~N})$ e AA $(1,39 \mathrm{~N})$, que não diferiram dos mantidos sob $\mathrm{AC} 1(1,58 \mathrm{~N})$. No $7^{\circ}$ dia de armazenamento a $5{ }^{\circ} \mathrm{C}$, os valores de firmeza dos frutos mantidos nas atmosferas AC1 $(1,38 \mathrm{~N}), \mathrm{AC} 2(1,62 \mathrm{~N})$ e AA $(1,11 \mathrm{~N})$ diferiram significativamente entre si $(\mathrm{p}<0,05)$. Os frutos mantidos sob $\mathrm{AA}$, a $5{ }^{\circ} \mathrm{C}$, foram os que apresentaram os menores valores médios de firmeza no período avaliado, diferindo de todos os demais tratamentos.

No $3^{\circ}$ dia de armazenamento, a $10^{\circ} \mathrm{C}$, não houve diferença significativa entre as atmosferas de armazenamento. Entretanto, no $7^{\circ}$ dia houve diferença significativa $(p<0,05)$ entre os valores de firmeza dos frutos mantidos nas atmosferas AC2 $(1,36 \mathrm{~N})$ e $\mathrm{AA}(1,15 \mathrm{~N})$, que não diferiram dos mantidos sob AC1 $(1,28)$.

Não houve variação significativa nos valores de firmeza ao longo do tempo de armazenamento nos frutos mantidos nas atmosferas $\mathrm{AC} 1$ e AC2, em ambas as temperaturas. Os valores de firmeza encontrados no $7^{\circ}$ dia para os morangos mantidos sob AA, em ambas as temperaturas, foram significativamente menores que no início do experimento.

Os resultados observados neste trabalho estão de acordo com os experimentos conduzidos por Brackmann et al. (2001). Estes autores verificaram o efeito da utilização de elevadas concentrações de $\mathrm{CO}_{2}$ no prolongamento da vida pós-colheita de morangos cv. Oso Grande in natura e observaram que, quanto maior a concentração de $\mathrm{CO}_{2}$ maior foi a firmeza da polpa dos frutos. $\mathrm{O}$ armazenamento sob atmosfera controlada reduz a atividade metabólica do tecido vegetal como um todo. Assim, ao reduzir a atividade metabólica global, contribui também para que seja reduzida a atividade das enzimas de metabolização da parede celular, o que em última análise faz com que o amolecimento dos tecidos ocorra de forma mais lenta (ROLLE; CHISM, 1987; WATADA; ABE; YAMUCHI, 1990).

Pressupõe-se que o aumento do valor de firmeza observado no $3^{\circ}$ dia de armazenamento na maioria dos tratamentos (Tabela 2), apesar de não significativo, pode ser devido à intensa desidratação dos frutos nos primeiros dias após o processamento mínimo, ou seja, até que ocorresse a completa cicatrização do tecido injuriado pelo corte. Esse ressecamento da epiderme dos frutos faz com que eles tenham maior resistência à penetração da ponteira teste, aumentando os valores de firmeza. A perda de firmeza pelos morangos processados minimamente, observada após 7 dias de armazenamento, pode ser efeito do processamento mínimo e do processo de senescência. Segundo Watada, Abe e Yamuchi (1990) e Rolle e Chism (1987), esse tipo de processamento aumenta a perecibilidade do produto, dado o aumento da atividade metabólica e da descompartimentalização de enzimas e substratos, podendo resultar em perda de firmeza e outras alterações. A perda de firmeza durante o armazenamento provavelmente está relacionada à hidrólise enzimática dos componentes da parede celular. Enzimas proteolíticas e pectinolíticas liberadas das células danificadas pelo corte tam-
Tabela 2. Variação do pH e da acidez total titulável em morangos processados minimamente e mantidos sob atmosfera controlada a $5 \mathrm{e}$ $10{ }^{\circ} \mathrm{C}$ e $85 \%$ UR.

\begin{tabular}{|c|c|c|c|c|c|c|}
\hline \multirow{4}{*}{$\begin{array}{c}\text { Tempo de } \\
\text { armazenamento } \\
\text { (dias) }\end{array}$} & \multicolumn{6}{|c|}{ Tratamentos } \\
\hline & \multicolumn{2}{|c|}{$\mathrm{AC} 1$} & \multicolumn{2}{|c|}{ AC2 } & \multicolumn{2}{|c|}{ AA } \\
\hline & $5^{\circ} \mathrm{C}$ & $10^{\circ} \mathrm{C}$ & $5^{\circ} \mathrm{C}$ & $10^{\circ} \mathrm{C}$ & $5^{\circ} \mathrm{C}$ & $10^{\circ} \mathrm{C}$ \\
\hline & \multicolumn{6}{|c|}{$\mathrm{pH}$} \\
\hline 0 & $3,71^{\mathrm{Ab}}$ & $3,71^{\mathrm{Ab}}$ & $3,71^{\mathrm{Ab}}$ & $3,71^{\mathrm{Ab}}$ & $3,71^{\mathrm{Ab}}$ & $3,71^{\mathrm{Aa}}$ \\
\hline 3 & $3,71^{\mathrm{ABb}}$ & $3,73^{\mathrm{Ab}}$ & $3,74^{\mathrm{Ab}}$ & $3,70^{\mathrm{Ab}}$ & $3,67^{\mathrm{Bb}}$ & $3,73^{\mathrm{Aa}}$ \\
\hline \multirow[t]{2}{*}{7} & $3,82^{\mathrm{ABa}}$ & $3,81^{\mathrm{ABa}}$ & $3,86^{\text {Aa }}$ & $3,86^{\mathrm{Aa}}$ & $3,81^{\mathrm{ABa}}$ & $3,77^{\mathrm{Ba}}$ \\
\hline & \multicolumn{6}{|c|}{ Acidez total titulável (mg ácido cítrico $100 \mathrm{~g}^{-1}$ ) } \\
\hline 0 & $0,60^{\mathrm{Aa}}$ & $0,60^{\mathrm{Aa}}$ & $0,60^{\mathrm{Aa}}$ & $0,60^{\mathrm{Aa}}$ & $0,60^{\mathrm{Aa}}$ & $0,60^{\text {Aa }}$ \\
\hline 3 & $0,59^{\mathrm{Aa}}$ & $0,54^{\mathrm{Bb}}$ & $0,56^{\mathrm{ABb}}$ & $0,61^{\mathrm{Aa}}$ & $0,60^{\mathrm{Aa}}$ & $0,58^{\mathrm{Aab}}$ \\
\hline 7 & $0,57^{\mathrm{ABa}}$ & $0,57^{\mathrm{ABb}}$ & $0,54^{\mathrm{Bb}}$ & $0,54^{\mathrm{Bb}}$ & $0,60^{\mathrm{Aa}}$ & $0,55^{\mathrm{Bb}}$ \\
\hline
\end{tabular}

Médias seguidas pela mesma letra, maiúscula na horizontal e minúscula na vertical, são estatisticamente iguais pelo teste de $\mathrm{LSD}$ a $5 \%$ de significância. $\mathrm{ACl}=3 \% \mathrm{O}_{2}+10 \% \mathrm{CO}_{2}$; $\mathrm{AC} 2=3 \% \mathrm{O}_{2}+15 \% \mathrm{CO}_{2}$; e $\mathrm{AA}=$ atmosfera ambiente (controle).

bém podem se difundir para os tecidos internos, provocando perda de firmeza.

\section{$3.3 p H$}

Foram observadas diferenças significativas somente para o fator tempo de armazenamento $(\mathrm{p}<0,05)$. A temperatura $\mathrm{e}$ a atmosfera de armazenamento, avaliadas de maneira isolada, não tiveram efeito significativo sobre o $\mathrm{pH}$ (Tabela 2). Houve aumento significativo do $\mathrm{pH}$ nos frutos de todos os tratamentos no $7^{\circ}$ dia de armazenamento, com exceção dos mantidos sob AA a $10^{\circ} \mathrm{C}$, nos quais esse aumento não foi significativo.

No $3^{\circ}$ dia de armazenamento, a $5{ }^{\circ} \mathrm{C}$, houve diferença significativa de $\mathrm{pH}$ entre os frutos mantidos nas atmosferas $\mathrm{AC} 2$ $(3,74)$ e AA $(3,67)$, que não diferiram dos mantidos sob AC1 $(3,71)$. Não houve diferença entre as atmosferas de armazenamento no $7^{\circ}$ dia de armazenamento, nesta mesma temperatura. A $10^{\circ} \mathrm{C}$ não houve diferença significativa entre as atmosferas no $3^{\circ}$ dia de armazenamento. Porém, no $7^{\circ}$ dia houve diferença significativa entre $\operatorname{AC} 2(3,86)$ e $\operatorname{AA}(3,77)(p<0,05)$, que não diferiram da $\mathrm{AC} 1(3,81)$.

Embora a diferença entre as atmosferas não tenha sido expressiva neste trabalho, diversos autores (GIL; HOLCROFT; KADER, 1997; HOLCROFT; KADER, 1999; KE et al., 1991; WRIGHT; KADER, 1997) comprovaram que altas concentrações de $\mathrm{CO}_{2}$ afetam o $\mathrm{pH}$ pela dissolução do gás $\mathrm{CO}_{2}$ e/ou pelo efeito da atmosfera controlada no metabolismo dos ácidos orgânicos.

\subsection{Acidez total titulável}

Foram observadas diferenças significativas para os fatores tempo de armazenamento e para as interações atmosferatemperatura e tempo-temperatura-atmosfera $(\mathrm{p}<0,05)$. A atmosfera e a temperatura de armazenamento, isoladas, também não influenciaram significativamente essa variável (Tabela 2).

Em geral, a acidez total titulável dos frutos diminuiu significativamente ao longo do período de armazenamento, com exceção dos mantidos em AA a $5{ }^{\circ} \mathrm{C}$, que mantiveram a acidez constante. Houve redução significativa da acidez titulável 
$(8,33 \%)$ no $7^{\text {o }}$ dia de armazenamento nos frutos mantidos sob AA quando a temperatura aumentou de $5{ }^{\circ} \mathrm{C}\left(0,60 \mathrm{mg} .100 \mathrm{~g}^{-1}\right)$ para $10^{\circ} \mathrm{C}\left(0,55 \mathrm{mg} \cdot 100 \mathrm{~g}^{-1}\right)$, não havendo diferença significativa entre os frutos mantidos sob AC1 e sob AC2.

Tanto no $3^{\circ}$ quanto no $7^{\circ}$ dia de armazenamento a $5^{\circ} \mathrm{C}$ houve diferença significativa entre os frutos mantidos sob as atmosferas AC2 e a AA, que não diferiram dos mantidos sob AC1. $\mathrm{No} 3^{\circ}$ dia de armazenamento a $10^{\circ} \mathrm{C}$ os frutos mantidos sob AC1 $\left(0,54 \mathrm{mg} .100 \mathrm{~g}^{-1}\right)$ diferiram significativamente dos mantidos nas atmosferas AC2 (0,61 mg.100 g $\left.\mathrm{g}^{-1}\right)$ e AA (0,58 mg.100 g $\left.\mathrm{g}^{-1}\right)$, que não diferiram significativamente entre si. No $7^{\circ}$ dia de armazenamento não houve diferenças entre as atmosferas.

A $5{ }^{\circ} \mathrm{C}$ somente os frutos mantidos sob AC2 tiveram perdas significativas na acidez total titulável ao longo do período de armazenamento, cerca de $10 \%$ do valor inicial. A $10{ }^{\circ} \mathrm{C}$, do dia zero para o $3^{\circ}$ dia de armazenamento, somente os frutos mantidos na AC1 tiveram redução significativa, cerca de $10 \%$. Do dia zero para o $7^{\circ}$ dia houve redução na acidez titulável dos frutos mantidos nas três atmosferas.

Os resultados encontrados estão de acordo com o esperado, pois os ácidos orgânicos tendem a diminuir durante o amadurecimento, em virtude de sua utilização como substrato para a respiração. Wright e Kader (1997) também observaram redução significativa da acidez titulável em morangos cv. Selva processados minimamente e armazenados por 7 dias a $5{ }^{\circ} \mathrm{C}$, tanto em atmosfera ambiente quanto em atmosferas com diferentes concentrações de $\mathrm{O}_{2}$ e $\mathrm{CO}_{2}$. Assim como no presente experimento, estes autores observaram que os frutos mantidos nas atmosferas contendo altas concentrações de $\mathrm{CO}_{2}$ foram os que tiveram maior redução na acidez titulável. Apesar do resultado encontrado neste trabalho estar de acordo com diversos autores, que também observaram aumento de $\mathrm{pH}$ e redução da acidez em morangos mantidos sob atmosfera controlada, existe a hipótese de que atmosferas contendo altas concentrações de $\mathrm{CO}_{2}$ podem interferir no $\mathrm{pH}$ intracelular de frutas e hortaliças, aumentando a acidez e reduzindo o $\mathrm{pH}$, por meio da hidratação do $\mathrm{CO}_{2}$ com produção de $\mathrm{HCO}_{3}{ }^{-}$e $\mathrm{H}^{+}$e/ou pelo seu efeito no metabolismo dos ácidos orgânicos.

Segundo Holcroft e Kader (1999), embora o aumento do pH em morangos armazenados possa ser devido ao efeito de uma ação externa, como em alface, a acidificação do vacúolo nesses frutos devido à dissociação do ácido carbônico é pouco provável. O acúmulo de ácidos fracos, como o cítrico e o málico, torna o vacúolo tamponado, enquanto o contrário é observado tanto em alface quanto em abacate. Enquanto a dissociação de ácido carbônico no citoplasma é passível de ocorrer, uma vez que o vacúolo é acidificado, a dissociação do ácido carbônico deve ser mínima e é provável que a capacidade tamponante do tecido possa absorver essas mudanças. Alternativamente, no $\mathrm{pH}$ vacuolar, bicarbonatos poderiam se formar e o $\mathrm{pH}$ poderia ser aumentado, mas essas mudanças deveriam ser também tamponadas.

\subsection{Sólidos solúveis}

Foi observada diferença significativa somente para a interação tempo-temperatura-atmosfera $(\mathrm{p}<0,05)$. A temperatura, a atmosfera e o tempo de armazenamento, de maneira isolada, não afetaram significativamente o teor de sólidos solúveis (Tabela 3).

Os únicos frutos cujos valores de sólidos solúveis encontrados diferiram significativamente dos valores encontrados no dia zero $\left(5,5^{\circ} \mathrm{Brix}\right)$ foram os armazenados na AA a $5^{\circ} \mathrm{C}$, avaliados no $3^{\circ}$ dia do armazenamento $\left(6,1^{\circ} \mathrm{Brix}\right)$. Neste dia esses frutos apresentaram teores significativamente superiores aos mantidos nas $\mathrm{AC} 1$ (5,5 $\left.{ }^{\circ} \mathrm{Brix}\right)$ e $\mathrm{AC} 2\left(5,27^{\circ} \mathrm{Brix}\right)$. No $7^{\circ}$ dia de armazenamento não foram observadas diferenças significativas entre as atmosferas, tanto a $5^{\circ} \mathrm{C}$ quanto a $10^{\circ} \mathrm{C}$.

Os resultados encontrados estão de acordo com Wright e Kader (1997), que estudando o comportamento de morangos cv. Selva processados minimamente e armazenados em atmosferas contendo diferentes concentrações de $\mathrm{O}_{2}$ e $\mathrm{CO}_{2}$, por 7 dias a $5{ }^{\circ} \mathrm{C}$, não observaram diferenças significativas nos teores de sólidos solúveis $(7,1$ a $8,6 \%)$ entre os tratamentos e ao longo do armazenamento.

A diferença significativa encontrada para a variável sólidos solúveis pode ser explicada pela variabilidade das amostras de morango, que apesar de terem sido selecionadas quanto à cor $\mathrm{e}$ tamanho no início do experimento, possivelmente apresentavam diferenças entre si.

\subsection{Teor de açúcares}

\section{Sacarose}

Não foi detectada presença de sacarose em nenhuma das amostras avaliadas. Na literatura há trabalhos que indicam que o teor de sacarose em morangos é em torno de $1 \%$ do total de açúcares. No presente trabalho, pode ter ocorrido uma hidrólise da sacarose contida nos frutos em glicose + frutose, no período entre a colheita e o processamento, sendo que os frutos encontravam-se maduros e foram resfriados somente 12 horas após a colheita. Nunes et al. (1995) observaram em experimentos com morangos reduções expressivas nos teores de açúcares (glicose, frutose e sacarose) nos frutos que tiveram uma demora de 6 horas para serem resfriados, em relação aos frutos que tiveram resfriamento imediato após a colheita.

Cordenunsi, Nascimento e Lajolo (2003) avaliaram as mudanças físico-químicas relacionadas à qualidade de cinco cultivares de morango durante armazenamento refrigerado $\left(6^{\circ} \mathrm{C}\right)$ e observaram que a sacarose desapareceu em todas elas

Tabela 3. Variação do teor de sólidos solúveis em morangos processados minimamente e mantidos sob atmosfera controlada a 5 e $10^{\circ} \mathrm{C}$ e $85 \%$ UR.

\begin{tabular}{|c|c|c|c|c|c|c|}
\hline \multirow{3}{*}{$\begin{array}{c}\text { Tempo de } \\
\text { armazenamento } \\
\text { (dias) }\end{array}$} & \multicolumn{6}{|c|}{ Sólidos solúveis ( $\left.{ }^{\circ} \mathrm{Brix}\right)$} \\
\hline & \multicolumn{2}{|c|}{$\mathrm{AC} 1$} & \multicolumn{2}{|c|}{$\mathrm{AC} 2$} & \multicolumn{2}{|c|}{ AA } \\
\hline & $5^{\circ} \mathrm{C}$ & $10^{\circ} \mathrm{C}$ & $5^{\circ} \mathrm{C}$ & $10^{\circ} \mathrm{C}$ & $5^{\circ} \mathrm{C}$ & $10^{\circ} \mathrm{C}$ \\
\hline 0 & $5,50^{\mathrm{Aa}}$ & $5,50^{\mathrm{Aa}}$ & $5,50^{\mathrm{Aa}}$ & $5,50^{\mathrm{Aab}}$ & $5,50^{\mathrm{Ab}}$ & $5,50^{\mathrm{Aa}}$ \\
\hline 3 & $5,52^{\mathrm{BCa}}$ & $5,43^{\mathrm{BCa}}$ & $5,27^{\mathrm{Ca}}$ & $5,82^{\mathrm{ABa}}$ & $6,13^{\mathrm{Aa}}$ & $5,58^{\mathrm{BCa}}$ \\
\hline 7 & $5,87^{\mathrm{Aa}}$ & $5,27^{\mathrm{Ba}}$ & $5,67^{\mathrm{ABa}}$ & $5,28^{\mathrm{Bb}}$ & $5,70^{\mathrm{ABab}}$ & $5,63^{\mathrm{ABa}}$ \\
\hline
\end{tabular}

Médias seguidas pela mesma letra, maiúscula na horizontal e minúscula na vertical, são estatisticamente iguais pelo teste de LSD a $5 \%$ de significância. AC1 $=3 \% \mathrm{O}_{2}+10 \% \mathrm{CO}_{2}$; $\mathrm{AC} 2=3 \% \mathrm{O}_{2}+15 \% \mathrm{CO}_{2} ; \mathrm{e} \mathrm{AA}=$ atmosfera ambiente (controle). 
no $2^{\circ}$ dia de armazenamento. Os mesmos autores propõem que a alta atividade metabólica dos frutos pode ter contribuído para o consumo de sacarose pelo tecido, já que o desaparecimento da sacarose não resultou em aumento nos teores de glicose e frutose na maioria das cultivares estudadas.

\section{Frutose}

Foram observadas diferenças significativas para os fatores atmosfera e tempo de armazenamento $(p<0,05)$. A temperatura de armazenamento, isolada, não afetou significativamente esta variável (Tabela 4 ).

No $3^{\circ}$ dia de armazenamento a $5^{\circ} \mathrm{C}$, foi observada diferença significativa para o fator atmosfera, no qual os frutos mantidos sob AA $\left(3,27 \mathrm{~g} .100 \mathrm{~g}^{-1}\right)$ apresentaram teor de frutose significativamente superior aos frutos mantidos nas demais atmosferas (2,14 e 2,15 g. $100 \mathrm{~g}^{-1}, \mathrm{AC} 1$ e AC2, respectivamente), que não diferiram entre si. No $7^{\circ}$ dia de armazenamento a $10^{\circ} \mathrm{C}$, os frutos mantidos nas atmosferas AA $\left(3,27\right.$ g. $\left.100 \mathrm{~g}^{-1}\right)$ e AC1 (2,52 g. $\left.100 \mathrm{~g}^{-1}\right)$ diferiram significativamente entre si, porém não diferiram dos mantidos sob AC2 (3,06 g.100 g $\left.\mathrm{g}^{-1}\right)$.

Em relação ao tempo de armazenamento, observa-se que houve redução significativa nos teores de frutose do dia zero (3,27 g. $\left.100 \mathrm{~g}^{-1}\right)$ para o $3^{\circ}$ dia de armazenamento em todos os tratamentos, em ambas as temperaturas, exceto nos frutos mantidos sob AA a $5{ }^{\circ} \mathrm{C}$, os quais mantiveram o teor deste açúcar constante $\left(3,27 \mathrm{~g} .100 \mathrm{~g}^{-1}\right)$. No $7^{\circ}$ dia de armazenamento só houve redução significativa nos teores de frutose em relação ao dia zero nos frutos mantidos sob AC1 e a $10^{\circ} \mathrm{C}\left(2,52\right.$ g. $\left.100 \mathrm{~g}^{-1}\right)$

\section{Glicose}

Foram observadas diferenças significativas para o fator tempo de armazenamento $(\mathrm{p}<0,05)$. A temperatura e a atmosfera de armazenamento, avaliadas de maneira isolada, não influenciaram significativamente os teores de glicose (Tabela 4).

De maneira geral, os teores de glicose no final do armazenamento ( $7^{\circ} \mathrm{dia}$ ) não diferiram significativamente dos

Tabela 4. Variação do teor de frutose e glicose em morangos processados minimamente e mantidos sob atmosfera controlada a 5 e $10^{\circ} \mathrm{C}$ e $85 \%$ UR.

\begin{tabular}{|c|c|c|c|c|c|c|}
\hline \multirow{4}{*}{$\begin{array}{c}\text { Tempo de } \\
\text { armazenamento } \\
\text { (dias) }\end{array}$} & \multicolumn{6}{|c|}{ Tratamentos } \\
\hline & \multicolumn{2}{|c|}{$\mathrm{AC1}$} & \multicolumn{2}{|c|}{$\mathrm{AC} 2$} & \multicolumn{2}{|c|}{ AA } \\
\hline & $5{ }^{\circ} \mathrm{C}$ & $10^{\circ} \mathrm{C}$ & $5^{\circ} \mathrm{C}$ & $10^{\circ} \mathrm{C}$ & $5^{\circ} \mathrm{C}$ & $10^{\circ} \mathrm{C}$ \\
\hline & \multicolumn{6}{|c|}{ Frutose $\left(\mathrm{g} .100 \mathrm{~g}^{-1}\right)$} \\
\hline 0 & $3,27^{\mathrm{Aa}}$ & $3,27^{\mathrm{Aa}}$ & $3,27^{\mathrm{Aa}}$ & $3,27^{\mathrm{Aa}}$ & $3,27^{\mathrm{Aa}}$ & $3,27^{\mathrm{Aa}}$ \\
\hline 3 & $2,14^{\mathrm{Bb}}$ & $1,91^{\mathrm{Bb}}$ & $2,15^{\mathrm{Bb}}$ & $1,98^{\mathrm{Bb}}$ & $3,27^{\mathrm{Aa}}$ & $2,02^{\mathrm{Bb}}$ \\
\hline & $2,90^{\mathrm{Ba}}$ & $2,52^{\mathrm{Bb}}$ & $2,66^{\mathrm{Bab}}$ & $3,06^{\mathrm{ABa}}$ & $3,19^{\mathrm{Aa}}$ & $3,27^{\mathrm{Aa}}$ \\
\hline & \multicolumn{6}{|c|}{ Glicose $\left(\mathrm{g} .100 \mathrm{~g}^{-1}\right)$} \\
\hline 0 & $2,31^{\mathrm{Aa}}$ & $2,31^{\mathrm{Aa}}$ & $2,31^{\mathrm{Aa}}$ & $2,31^{\mathrm{Aa}}$ & $2,31^{\mathrm{Aa}}$ & $2,31^{\mathrm{Ab}}$ \\
\hline 3 & $1,51^{\mathrm{Aa}}$ & $1,64^{\mathrm{Aa}}$ & $1,61^{\mathrm{Aa}}$ & $1,68^{\mathrm{Aa}}$ & $2,23^{\mathrm{Aa}}$ & $1,50^{\mathrm{Ab}}$ \\
\hline 7 & $2,33^{\mathrm{Ba}}$ & $2,24^{\mathrm{Ba}}$ & $2,81^{\mathrm{ABa}}$ & $2,03^{\mathrm{Ba}}$ & $2,80^{\mathrm{ABa}}$ & $3,53^{\mathrm{Aa}}$ \\
\hline
\end{tabular}

Médias seguidas pela mesma letra, maiúscula na horizontal e minúscula na vertical, são estatisticamente iguais pelo teste de LSD a 5\% de significância. $\mathrm{ACl}=3 \% \mathrm{O}_{2}+10 \% \mathrm{CO}_{2}$; $\mathrm{AC} 2=3 \% \mathrm{O}_{2}+15 \% \mathrm{CO}_{2} ;$ e AA = atmosfera ambiente (controle). encontrados no início $\left(2,31 \mathrm{~g} .100 \mathrm{~g}^{-1}\right)$, com exceção dos frutos mantidos em AA a $10{ }^{\circ} \mathrm{C}$, que apresentaram teor de glicose significativamente superior ao valor inicial $\left(3,53 \mathrm{~g} .100 \mathrm{~g}^{-1}\right)$. No $3^{\circ}$ dia de armazenamento, tanto a $5^{\circ} \mathrm{C}$ quanto a $10{ }^{\circ} \mathrm{C}$, houve redução nos teores de glicose em todos os tratamentos, porém não significativamente. Não houve diferença significativa no $3^{\circ}$ dia de armazenamento entre os frutos mantidos sob AA a 5 e a $10{ }^{\circ} \mathrm{C}\left(2,23\right.$ e $1,50 \mathrm{~g} .100 \mathrm{~g}^{-1}$, respectivamente $)$. No $3^{\circ}$ dia de armazenamento não houve diferença significativa entre as atmosferas, tanto a $5^{\circ} \mathrm{C}$ quanto a $10^{\circ} \mathrm{C}$. No $7^{\circ}$ dia, a $5^{\circ} \mathrm{C}$, também não foram observadas diferenças significativas entre as atmosferas. Porém, neste mesmo dia a $10^{\circ} \mathrm{C}$, o teor de glicose dos frutos mantidos na AA $\left(3,53 \mathrm{~g} .100 \mathrm{~g}^{-1}\right)$ foi significativamente superior ao dos frutos mantidos nas atmosferas AC1 e AC2 (2,24 e 2,03 g.100 g $\mathrm{g}^{-1}$, respectivamente).

O fato dos teores de glicose e de frutose, na maioria dos tratamentos, terem diminuído no $3^{\circ}$ dia de armazenamento, e de no $7^{\circ}$ dia apresentarem valores próximos aos iniciais, pode ser devido ao aumento no metabolismo dos frutos logo após o processamento mínimo, causado pelos estresses físicos aos quais foram submetidos, aumentando a taxa respiratória e fazendo com que ocorresse uma queda dos teores de glicose e frutose nos primeiros dias do armazenamento, até estabelecer-se o equilíbrio. $\mathrm{O}$ aumento no teor destes açúcares, observado no $7^{\circ} \mathrm{dia}$, pode estar relacionado, em parte, à concentração dos mesmos no tecido vegetal, provocado pela perda de água observada nos frutos durante o armazenamento, principalmente nos frutos mantidos em atmosfera ambiente.

Pelayo, Ebeler e Kader (2003) não observaram reduções significativas nos teores de glicose e de frutose ao longo do armazenamento de morangos das cultivares Aroma, Diamante e Selva armazenadas em atmosfera ambiente e em atmosfera ambiente $+20 \% \mathrm{CO}_{2}$, a $5{ }^{\circ} \mathrm{C}$. Estes autores também não observaram diferenças significativas entre as atmosferas de armazenamento, exceto no $11^{\circ}$ e no $13^{\circ}$ dias para morangos cv. Selva, cuja concentração de frutose foi significativamente menor nos frutos mantidos em atmosfera ambiente $+20 \% \mathrm{CO}_{2}$, que nos mantidos em atmosfera ambiente, o que também foi constatado neste experimento com morangos cv. Oso Grande processados minimamente.

\subsection{Antocianinas totais}

Foram observadas diferenças significativas somente para atmosfera de armazenamento $(\mathrm{p}<0,05)$. A temperatura e o tempo de armazenamento, avaliados isoladamente, não afetaram o teor de antocianinas totais (Tabela 5).

A variação verificada nos teores de antocianinas em função da alteração da atmosfera de armazenamento demonstra a influência dos elevados níveis de $\mathrm{CO}_{2}$ no metabolismo dos pigmentos de morangos processados minimamente. Adicionalmente, as alterações verificadas no $\mathrm{pH}$ dos frutos, como visto anteriormente, também podem influenciar o metabolismo e a estabilidade desses pigmentos, ocasionando perdas significativas de cor. Trabalhos realizados por Holcroft e Kader (1999) indicam que há relação direta entre a concentração de $\mathrm{CO}_{2}$ e a redução na síntese de antocianinas. 
Tabela 5. Variação do teor de antocianinas totais em morangos processados minimamente e mantidos sob atmosfera controlada a 5 e $10{ }^{\circ} \mathrm{C}$ e $85 \%$ UR.

\begin{tabular}{|c|c|c|c|c|c|c|}
\hline \multirow{3}{*}{$\begin{array}{c}\text { Tempo de } \\
\text { armazenamento } \\
\text { (dias) }\end{array}$} & \multicolumn{6}{|c|}{ Antocianinas totais (mg.100 g $\mathrm{g}^{-1}$ ) } \\
\hline & \multicolumn{2}{|c|}{$\mathrm{AC} 1$} & \multicolumn{2}{|c|}{$\mathrm{AC} 2$} & \multicolumn{2}{|c|}{ AA } \\
\hline & $5^{\circ} \mathrm{C}$ & $10^{\circ} \mathrm{C}$ & $5^{\circ} \mathrm{C}$ & $10^{\circ} \mathrm{C}$ & $5^{\circ} \mathrm{C}$ & $10^{\circ} \mathrm{C}$ \\
\hline 0 & $21,83^{\mathrm{Aa}}$ & $21,83^{\mathrm{Aa}}$ & $21,83^{\mathrm{Aa}}$ & $21,83^{\mathrm{Aa}}$ & $21,83^{\mathrm{Aa}}$ & $21,83^{\mathrm{Aa}}$ \\
\hline 3 & $22,26^{\mathrm{ABa}}$ & $20,85^{\mathrm{Ba}}$ & $19,93^{\mathrm{Ba}}$ & $19,69^{\mathrm{Ba}}$ & $24,24^{\mathrm{Aa}}$ & $22,60^{\mathrm{ABa}}$ \\
\hline 7 & $21,43^{\mathrm{ABa}}$ & $19,14^{\mathrm{Ba}}$ & $22,17^{\mathrm{ABa}}$ & $20,13^{\mathrm{Ba}}$ & $23,83^{\mathrm{Aa}}$ & $24,68^{\mathrm{Aa}}$ \\
\hline
\end{tabular}

Médias seguidas pela mesma letra, maiúscula na horizontal e minúscula na vertical, são estatisticamente iguais pelo teste de LSD a 5\% de significância. AC1 $=3 \% \mathrm{O}_{2}+10 \% \mathrm{CO}_{2}$; $\mathrm{AC} 2=3 \% \mathrm{O}_{2}+15 \% \mathrm{CO}_{2}$; e AA = atmosfera ambiente (controle).

No $3^{\circ}$ dia de armazenamento, a $5^{\circ} \mathrm{C}$, houve diferença significativa entre os frutos mantidos sob AA (24,24 mg.100 g $\left.\mathrm{g}^{-1}\right)$ e sob AC2 (19,93 mg.100 g $\left.{ }^{-1}\right)$. No $7^{\circ}$ dia de armazenamento, nesta mesma temperatura, não houve diferença significativa entre as atmosferas. No $3^{\circ}$ dia de armazenamento, a $10^{\circ} \mathrm{C}$, não houve diferença significativa entre as atmosferas. Porém no $7^{\circ}$ dia, nesta mesma temperatura, os frutos mantidos sob AA (24,68 mg.100 g $\left.\mathrm{g}^{-1}\right)$ diferiram significativamente das atmosferas AC1 (19,14 mg.100 g $\left.{ }^{-1}\right)$ e AC2 (20,13 mg.100 g $\left.{ }^{-1}\right)$, que não diferiram entre si. Não houve variação significativa no teor de antocianinas dos frutos ao longo do armazenamento em nenhuma das atmosferas, tanto a $5{ }^{\circ} \mathrm{C}$ quanto a $10^{\circ} \mathrm{C}$. Em geral, os frutos armazenados sob AA apresentaram teor de antocianinas totais superior aos armazenados nas atmosferas AC1 e AC2 (Figura 2d).

Os resultados encontrados estão de acordo com Holcroft e Kader (1999), que observaram que os tecidos externos de morangos armazenados sob atmosfera ambiente e sob $2 \% \mathrm{O}_{2}$ (balanço $\mathrm{N}_{2}$ ) apresentaram maiores teores de antocianinas, após 5 e 10 dias, que os armazenados sob $0,5 \% \mathrm{O}_{2}\left(\right.$ balanço $\left.\mathrm{N}_{2}\right)$ e sob as atmosferas contendo altas concentrações de $\mathrm{CO}_{2}$.

Não houve influência do tempo de armazenamento no teor de antocianinas totais dos frutos processados minimamente, $o$ que está de acordo com Gil, Holcroft e Kader (1997), que também não encontraram diferenças significativas entre o teor de antocianinas inicial $\left(120,2 \mu \mathrm{g} \cdot \mathrm{g}^{-1}\right)$ e os valores obtidos a partir dos frutos armazenados.

\section{Conclusões}

Nas condições experimentais deste trabalho, verificou-se que o aumento da temperatura, de $5^{\circ} \mathrm{C}$ para $10^{\circ} \mathrm{C}$, não influenciou de forma significativa as variáveis físicas e químicas avaliadas. A utilização das atmosferas contendo $3 \% \mathrm{O}_{2}+10 \% \mathrm{CO}_{2}$ (balanço $\mathrm{N}_{2}$ ) e $3 \% \mathrm{O}_{2}+15 \% \mathrm{CO}_{2}$ (balanço $\mathrm{N}_{2}$ ) foi importante para a manutenção da qualidade dos morangos processados minimamente, pois estes mantiveram melhor firmeza em relação à atmosfera ambiente, e as atmosferas foram mais efetivas no controle da perda de massa dos frutos. Todavia, os frutos processados minimamente mantidos nestas atmosferas apresentaram menor teor de antocianinas que os mantidos em atmosfera ambiente.

\section{Agradecimentos}

À Embrapa Agroindústria de Alimentos (CTAA), por ter possibilitado a realização dos experimentos, à CAPES pelo consentimento da bolsa de estudos e ao CNPq pelo financiamento do projeto de pesquisa.

\section{Referências bibliográficas}

AGAR, I. T.; STREIF, J.; BANGERTH, F. Effect of high $\mathrm{CO}_{2}$ and controlled atmosphere (CA) on the ascorbic and dehydroascorbic acid content of some berry fruits. Postharvest Biology and Technology, Amsterdan, v. 11, n. 1, p. 47-55, 1997.

BRACKETT, R. E. Microbiological consequences of minimally processed fruits and vegetables. Journal of Food Quality, Trumbull, v. 10, n.3, p. 195-206, 1987.

BRACKMANN, A. et al. Armazenamento de morangos cv. Oso Grande (Fragaria ananassa L.) sob elevadas pressões de $\mathrm{CO}_{2}$. Revista Brasileira de Agrociência, Pelotas, v. 7, n. 1, p. 10-14, 2001.

BRECHT, J. K. et al. Maintaining optimal atmosphere conditions for fruits and vegetables throughout the postharvest handling chain. Postharvest Biology and Technology, Amsterdan, v. 27, n. 1, p. $87-101,2003$.

CORDENUNSI, B. R.; NASCIMENTO, J. R. O.; LAJOLO, F. M. Physico-chemical changes related to quality of five strawberry fruit cultivars during cool-storage. Food Chemistry, New York, v. 83, n. 2, p. 167-173, 2003.

DAMASCENO, K. S. F. da S. C.; STAMFORD, T. L. M.; ALVES, M. A. Vegetais minimamente processados: uma revisão. Higiene Alimentar, São Paulo, v. 15, n. 85, p. 20-25, 2001.

FULEKI, T.; FRANCIS, F. J. Quantitative methods for anthocyanins. 1. Extraction and determination of total anthocyanins in cranberries. Journal of Food Science, Chicago, v. 33, n. 1, p. 72-77, 1968.

GIL, M. I.; HOLCROFT, D. M.; KADER, A. A. Changes in strawberry anthocyanins and other polyphenols in response to carbon dioxide treatments. Journal of Agricultural and Food Chemistry, Washington, v. 45, n. 5, p. 1662-1667, 1997.

HOLCROFT, D. M.; KADER, A. A. Controlled atmosphere-induced changes in $\mathrm{pH}$ and organic acid metabolism may affect color of stored strawberry fruit. Postharvest Biology and Technology, Amsterdan, v. 17, n. 1, p. 19-32, 1999.

HONÓRIO, S. L.; MORETTI, C. L. Fisiologia pós-colheita de frutas e hortaliças. In: CORTEZ, L. A. B.; HONÓRIO, S. L; MORETTI, C. L. (Ed.) Resfriamento de frutas e hortaliças. Brasília, DF: Embrapa Informação Tecnológica, 2001. 428p.

INTERNATIONAL FRESH-CUT PRODUCE ASSOCIATION. Freshcut produce handling guidelines. 3 ed. Newark: Produce Marketing Association, 1999. $39 \mathrm{p}$.

ISO 750: fruit and vegetable products: determination of titratable acidity. 2 ed. Genève: International Organization for Standardization, 1998.

ISO 1842: fruit and vegetable products: determination of $\mathrm{pH} .2 \mathrm{ed}$. Genève: International Organization for Standardization, 1991.

ISO 2173: fruit and vegetable products: determination of soluble solids content: refractometric method. 1 ed. Genève: International Organization for Standardization, 1978.

KE, D.; GOLDSTEIN, L.; O’MAHONY, M.; KADER, A. A. Effects of short term exposure to low $\mathrm{O}_{2}$ atmospheres on quality attributes of strawberries. Journal of Food Science, Chicago, v. 56, n. 1, p. 50-54, 1991. 
LANA, M. M.; FINGER, F. L. Atmosfera modificada e controlada. Brasília, DF: Embrapa Comunicação para Transferência de Tecnologia: Embrapa Hortaliças, 2000. 34p.

MACRAE, R. Food science and technology: a series of monoghraphys: HPLC in food analysis. 2 ed. New York: Academic Press, 1998. $77 \mathrm{p}$.

NUNES, M. C. N. et al. Physical and chemical quality characteristics of strawberries after storage are reduced by a short delay to cooling. Postharvest Biology and Technology, Amsterdan, v. 6, n. 1-2, p. 17-28, 1995.

PELAYO, C.; EBELER, S. E.; KADER, A. A. Postharvest life and flavor quality of three strawberry cultivars kept at $5{ }^{\circ} \mathrm{C}$ in air $+20 \mathrm{KPa}$ $\mathrm{CO}_{2}$. Postharvest Biology and Technology, Amsterdan, v. 27, n. 2 , p. 171-183, 2003.

ROLLE, R.; CHISM, G. W. Physiological consequences of minimally processed fruits and vegetables. Journal of Food Quality, Trumbull, v. 10, n. 3, p. 157-177, 1987.

RONQUE, E. R. V. A cultura do morangueiro. Curitiba: EMATERPR, 1998. p. 183-202.
SISTRUNK, W. A.; MORRIS, J. R. Storage stability of strawberry products manufactured from mechanically-harvest strawberries. Journal of American Society of Horticultural Science, Alexandria, v. 103 , n. 5, p. 616-620, 1978.

SOLIVA-FORTUNY, R. C.; MARTÍN-BELLOSO, O. New advances in extending the shelf-life of fresh-cut fruits: a review. Trends in Food Science \& Technology, Oxford, v. 14, n. 9, p. 341-353, 2003.

SPAYD, S. E.; MORRIS, J. R. Effects of immature fruit and holding on strawberry puree quality and color stability. Journal of American Society of Horticultural Science, Alexandria, v. 106, n. 2, p. 211-216, 1981.

WATADA, A. E.; ABE, K.; YAMUCHI, N. Physiological activities of partially processed fruits and vegetables. Food Technology, Chicago, v. 44, n. 5, p. 116-122, 1990.

WRIGHT, K. P.; KADER, A. A. Effect of slicing and controlledatmosphere storage on the ascorbate content and quality of strawberries and persimmons. Postharvest Biology and Technology, Amsterdan, v. 10, n. 1, p. 39-48, 1997. 